\title{
呋咱并[3,4-e]-4,6-二氧化-1,2,3,4-四嗪新法合成与表征
}

\author{
李祥志王伯周* 李 辉 李亚南 \\ 毕福强霍 欢訤学忠 \\ (西安近代化学研究所 西安 710065)
}

\begin{abstract}
摘要 自行设计了呋咱并[3,4-e]-1,2,3,4-四嗪-4,6-二氧化(FTDO)新的合成路线, 采用 3,4-二氨基呋咱(DAF)为起始原料, 经过氧化、缩合、硝化、成环 4 步反应合成了 FTDO, 总收率为 $30.89 \%$, 并采用核磁共振光谱、红外光谱、质谱及元 素分析等进行了结构表征. 探讨了成环反应的机理, 并研究了硝化及成环反应条件, 确定硝化反应的最佳条件为: 硝 化试剂为 $100 \% \mathrm{HNO}_{3}, 25{ }^{\circ} \mathrm{C}$ 下反应 $2 \mathrm{~h}$, 收率为 $99.54 \%$; 成环反应的最佳条件为: $55{ }^{\circ} \mathrm{C}$ 下应 $5 \mathrm{~h}, n\left(\right.$ 化合物 4) : $n\left(\mathrm{P}_{2} \mathrm{O}_{5}\right)$ 为 $1: 16$, 溶剂量为 $160 \mathrm{~mL} / \mathrm{g}$, 收率为 $46.37 \%$.
\end{abstract}

关键词 呋咱并 $[3,4-e]-1,2,3,4$-四嗪-4,6-二氧化; 合成; 表征

\section{Novel Synthetic Route and Characterization of [1,2,5]oxadiazolo- $[3,4-e][1,2,3,4]$ tetrazine 4,6-Di-N-oxide (FTDO)}

\author{
Li, Xiangzhi Wang, Bozhou* Li, Hui Li, Yanan \\ Bi, Fuqiang Huo, Huan Fan, Xuezhong \\ (Xi'an Modern Chemistry Research Institute, Xi'an 710065)
}

\begin{abstract}
A novel synthetic route of $[1,2,5]$ oxadiazolo[3,4-e][1,2,3,4]tetrazine 4,6-di- $N$-oxide (FTDO) was designed and FTDO was synthesized from 3,4-diaminofurazan (DAF) via the reactions of oxidation, condensation, nitration and cyclization with a total yield of $30.89 \%$. FTDO and its intermediates were characterized by NMR, IR, MS and elemental analysis etc. The mechanism of cyclization was proposed and the conditions for the reaction of nitration and cyclization were optimized. It was found that the optimal conditions for the reaction of nitration were followed as: nitration reagent was $100 \% \mathrm{HNO}_{3}$, the reaction temperature was at $25{ }^{\circ} \mathrm{C}$ for $2 \mathrm{~h}$ with a yield of $99.54 \%$. The optimal conditions for the reaction of cyclization were followed as: the reaction temperature was at $55{ }^{\circ} \mathrm{C}$, the molar ratio of compound 4 and $\mathrm{P}_{2} \mathrm{O}_{5}$ was $1: 16$, and the volume of solvent was $160 \mathrm{~mL} / \mathrm{g}$ with a yield of $46.37 \%$.
\end{abstract}

Keyword $[1,2,5]$ oxadiazolo[3,4-e $][1,2,3,4]$ tetrazine 4,6-di- $N$-oxide; synthesis; characterization

高能量密度材料是当今含能材料领域研究的热点, 氮杂环类化合物因具有高生成焓、高密度、高氧平衡 等优点, 已经成为该领域备受关注的研究方向之一. 与 传统的含能材料相比, 1,3-二氧化-1,2,3,4-四嗪环是一种 结构新䓉的芳杂环 ${ }^{[1 \sim 6]}$, 将此类化合物引入推进剂配方 中, 能显著降低燃温及特征信号 ${ }^{[4,5,7,8]}$. 呋咱并 $[3,4-e]-$ 4,6-二氧化-1,2,3,4-四嗪 (FTDO) $)^{[9]}$ 是目前合成出的 1,2,3,4-四嗪-1,3-二氧化物中最有应用前景的含能化合 物, 其实测密度为 $1.85 \mathrm{~g} \cdot \mathrm{cm}^{-3}[10]$, 生成焓为 $4.23 \mathrm{MJ}$ • $\mathrm{kg}^{-1[11]}$, 计算爆速和爆压分别为 $9802 \mathrm{~m} \cdot \mathrm{s}^{-1}$ 和 44.78
$\mathrm{GPa}$ ，是一种具有潜在应用价值的含能材料，既可作为 火箭推进剂的氧化组分, 也可作为燃气发生剂使用 ${ }^{[12]}$. 国外报道了 FTDO 的 5 步合成方法 ${ }^{[9,13,14,15]}$, 总收率为 $14.63 \%$, 以二氨基呋咱为原料经过氧化、保护氨基、

缩合、去保护、成环得到 FTDO, 合成路线如 Scheme 1 所示. 该路线反应步骤较长, 过程繁琐, 格氏试剂需要 在无水无氧条件下制备使用; 3-氨基-4-硝基呋咱中的氨 基需要用 $N, N$-双(三甲基硅烷)胺保护、去保护、产物分 离, 提纯比较困难; 并且使用了较为昂贵的四氟硼酸硝 酰试剂, 无工业化应用前景.

\footnotetext{
*E-mail:wbz600@163.com

Received May 16, 2012; revised June 5, 2012; published online June 21, 2012.

Project supported by the National Defense Fundamental Scientific Research (No. B09201100051).

国防基础科研计划(No. B0920110051)资助项目.
} 


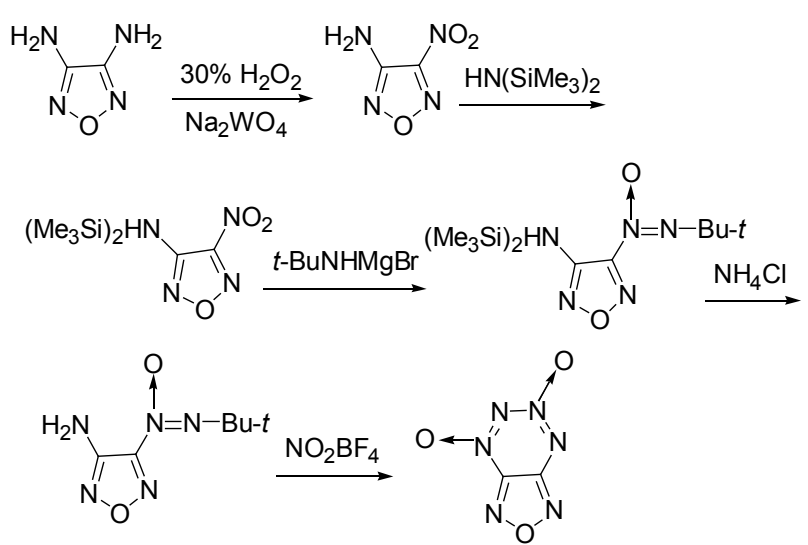

Scheme 1

针对上述合成路线的缺点, 参考文献 [16 19], 本 文设计了 FTDO 新的合成路线. 以 3,4-二氨基呋咱(1)为 起始原料, 苯为反应介质, 经 Caro's 酸氧化得到 3-氨 基-4-亚硝基呋咱(2), 然后与 $N, N$-二溴-叔丁基胺缩合得 到 3-氨基-4-(叔丁基氧化偶氮基)呋咱(3), 再用 100\%硝 酸硝化得到 3-硝氨基-4-(叔丁基氧化偶氮基)呋咱(4), 最 后用 $\mathrm{P}_{2} \mathrm{O}_{5}$ 成环成功地获得了 FTDO (5) 的高纯样品 (Scheme 2), 并通过 ${ }^{13} \mathrm{C} N M R,{ }^{14} \mathrm{~N} N M R,{ }^{15} \mathrm{~N} N M R,{ }^{17} \mathrm{O}$ NMR, IR, MS 和元素分析对标题化合物及其中间体结 构进行了表征. 该方法与文献报道的方法相比, 具有反 应步骤较少, 反应条件温和, 产物易于分离和提纯, 试 剂廉价易得等优点, 具有潜在的工业应用前景.
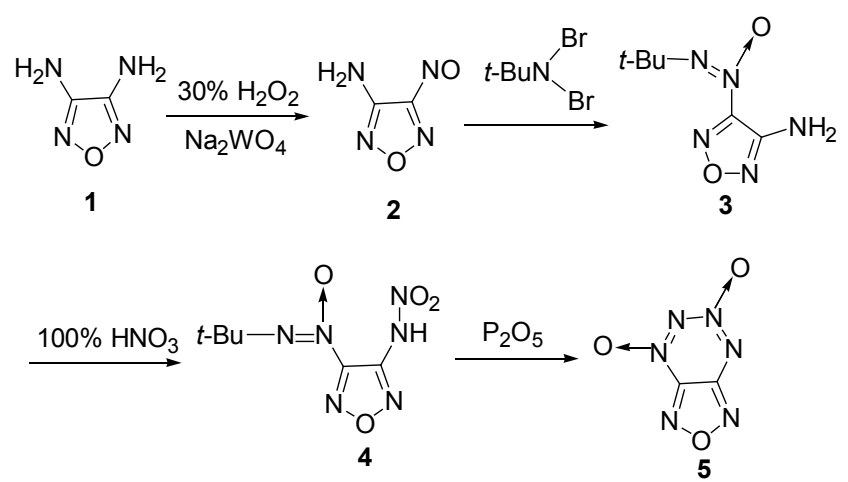

\section{Scheme 2}

\section{1 结果与讨论}

\section{1 成环反应机理的探讨}

化合物 4 存在共振式结构 $\mathbf{6}$, 当 $\mathrm{P}_{2} \mathrm{O}_{5}$ 存在时, $N$-羊弪 基与 $\mathrm{P}_{2} \mathrm{O}_{5}$ 发生酯化反应, 生成 3-(叔丁基氧化偶氮 基)-4-(偏磷酸偶氮氧化脂)呋咱(7), 然后 N-5 原子进攻 N-6 原子，与此同时脱去偏磷酸脂基，得到化合物 8 , 最 后脱去叔丁基得到目标产物 FTDO (5), 反应机理如 Scheme 3.

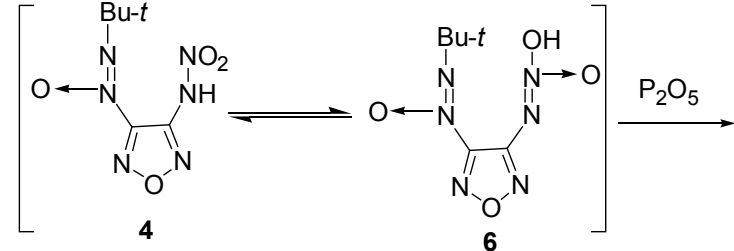

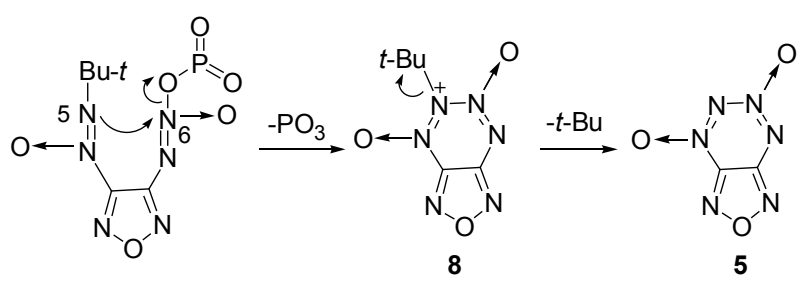

Scheme 3

\subsection{FTDO 的谱学解析}

\subsection{1 红外吸收光谱(IR)分析}

在 FTDO 红外吸收光谱图中，1624，1589，1514, $1460 \mathrm{~cm}^{-1}$ 为芳环双键的伸缩振动吸收, 表明分子结构 中有呋咱环; $1543,1418 \mathrm{~cm}^{-1}$ 为四嗪环双键的伸缩振动 吸收; $3000 \mathrm{~cm}^{-1}$ 附近无吸收，说明分子结构中没有 $\mathrm{C}-$ $\mathrm{H}, \mathrm{N}-\mathrm{H}$ 键, 与 FTDO 分子结构相符 ${ }^{[20]}$.

\subsection{2 核磁共振(NMR)分析}

图 1 4 分别为 FTDO 样品的 ${ }^{13} \mathrm{C}$ NMR 谱, ${ }^{14} \mathrm{~N}$ NMR 谱, ${ }^{15} \mathrm{~N}$ NMR 谱, ${ }^{17} \mathrm{O}$ NMR 谱. FTDO 样品的 ${ }^{1} \mathrm{H}$ NMR 谱 中, 除去気代氯仿溶剂残留峰, TMS 外无其他信号, 证 明该样品不含氢，与预定的结构式一致. ${ }^{13} \mathrm{C} \mathrm{NMR}$ 谱中 显示有两个峰, 分别为 $\delta 156.8,144.7$, 其碳原子数及化 学位移与文献报道相一致. ${ }^{14} \mathrm{~N}$ NMR 谱中显示有 5 个峰, 分别为 $\delta 37.6,10.0,-42.2,-50.7,-104.7 ;{ }^{15} \mathrm{~N}$ NMR 谱中有 5 个峰, 分别为 $\delta 35.6,38.8,-42.3,-50.9$, - 104.7; 综合两种氮谱，可以判断该分子中有六个氮原 子，其氮原子数及化学位移与文献报道一致; ${ }^{17} \mathrm{O} \mathrm{NMR}$ 谱中有 3 个峰, 分别为 $\delta 532.3,504.2,441.7$, 其氧原子 数及化学位移与文献报道一致 ${ }^{[9]}$.

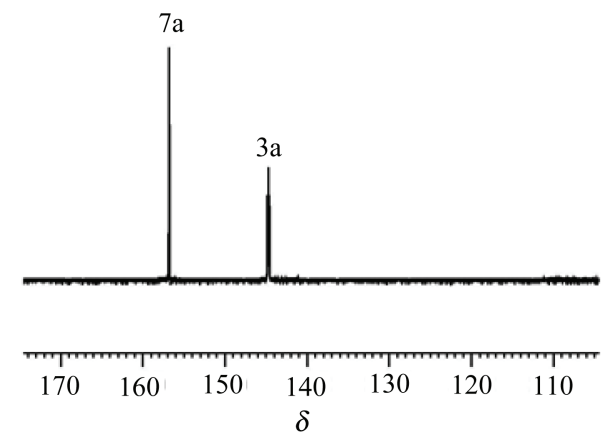

图 1 FTDO 的 ${ }^{13} \mathrm{C}$ NMR 图谱(acetone- $d_{6}, 25{ }^{\circ} \mathrm{C}$ ) Figure $1{ }^{13} \mathrm{C}$ NMR spectra of FTDO (acetone- $d_{6}, 25{ }^{\circ} \mathrm{C}$ ) 


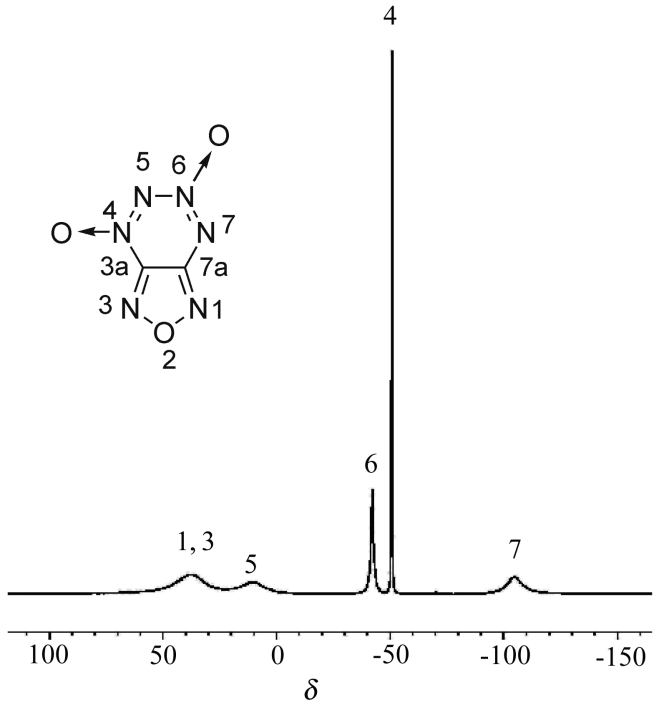

图 2 FTDO 的 ${ }^{14} \mathrm{~N}$ NMR 图谱(acetone- $d_{6}, 25{ }^{\circ} \mathrm{C}$ )

Figure $2{ }^{14} \mathrm{~N}$ NMR spectra of FTDO (acetone- $d_{6}, 25{ }^{\circ} \mathrm{C}$ )

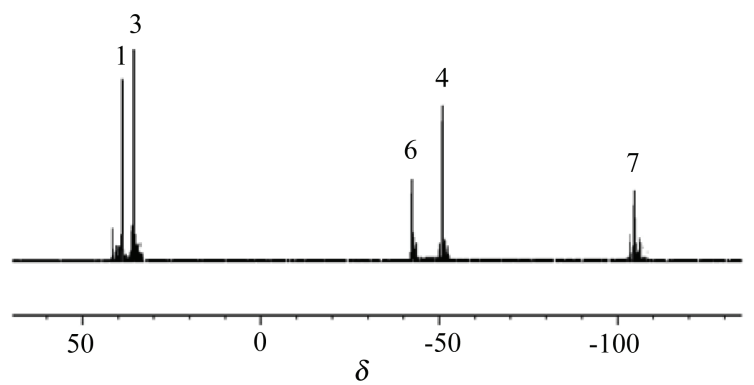

图 3 FTDO 的 ${ }^{15} \mathrm{~N}$ NMR 图谱(acetone- $d_{6}, 25{ }^{\circ} \mathrm{C}$ )

Figure $3{ }^{15} \mathrm{~N}$ NMR spectra of FTDO (acetone- $d_{6}, 25{ }^{\circ} \mathrm{C}$ )

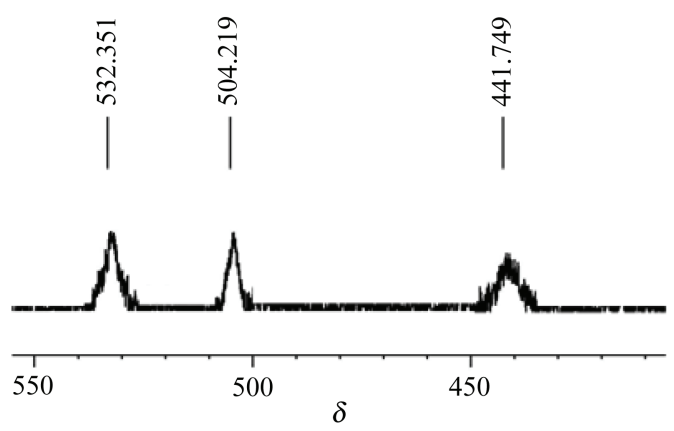

图 4 FTDO 的 ${ }^{17} \mathrm{ONMR}$ 图谱(acetone- $d_{6}, 25{ }^{\circ} \mathrm{C}$ )

Figure $4{ }^{17} \mathrm{O}$ NMR spectra of FTDO (acetone- $d_{6}, 25{ }^{\circ} \mathrm{C}$ )

\subsection{3 质谱(MS)解析}

FTDO 电离源(EI)质谱解析图谱如图 5 所示. 质谱 中的 $\mathrm{m} / \mathrm{z} 156$ 为 FTDO 的分子离子峰, 这与给物质的分 子量吻合，分子量为偶数，与分子中含有偶数个氮原子 一致; $m / z 84$ 碎片离子峰为 FTDO 分子裂解失去一分子 $\mathrm{N}_{2}$ 和一分子 $\mathrm{N}_{2} \mathrm{O}$ 的碎片峰; 进一步失去一分子 $\mathrm{O}$ 自由 基得到 $m / z 68$ 碎片离子; 或者由 FTDO 分子裂解失去两 分子 $\mathrm{N}_{2} \mathrm{O}$ 直接得到 $m / z 68$ 碎片离子; $m / z 68$ 碎片离子失
去一分子 $\mathrm{O}$ 自由基得到 $m / z 52$ 碎片离子, 进一步失去一 分子 $\mathrm{N}_{2}$ 得到 $m / z 24$ 碎片离子; $m / z 84$ 碎片离子失去两分 子 $\mathrm{NO}$ 也形成 $\mathrm{m} / \mathrm{z} 24$ 碎片离子, 同时产生 $\mathrm{m} / \mathrm{z} 30$ 的碎片 离子 ${ }^{[9,20]}$; 其可能的裂解过程见 Scheme 4 .

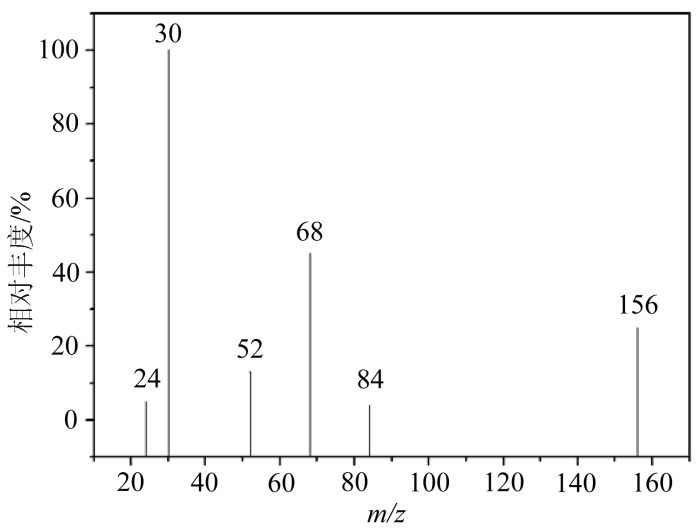

图 5 FTDO 的质谱图谱(EI)

Figure 5 MS spectra of FTDO(EI)

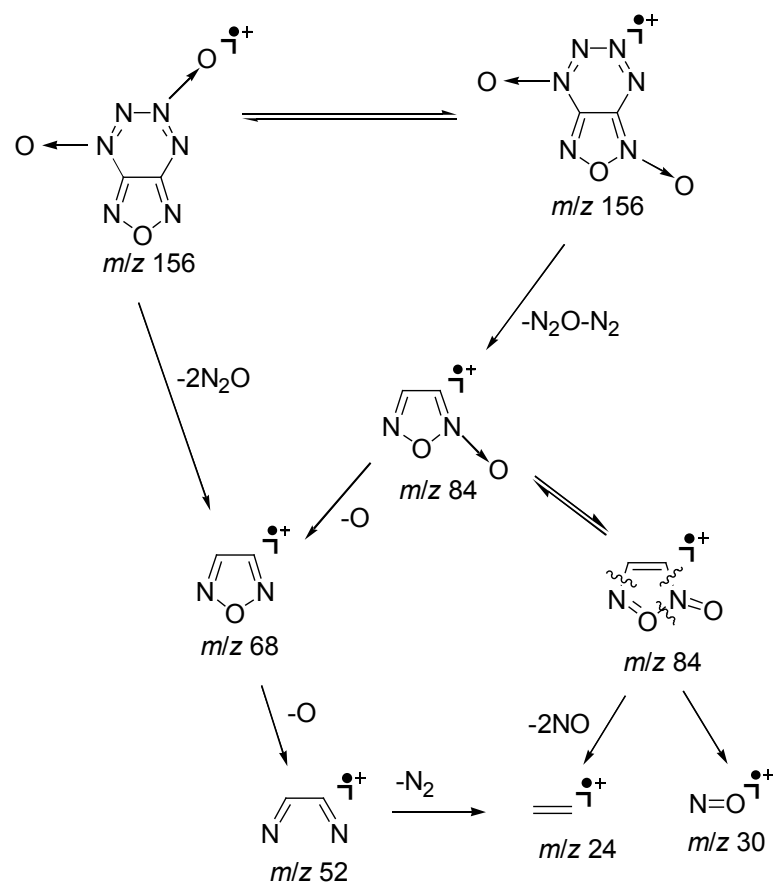

Scheme 4

\section{3 合成化合物 4 中硝化试剂的选择}

设定反应温度为 $25^{\circ} \mathrm{C}$, 反应时间为 $2 \mathrm{~h}$, 考察了醋 酐/硝酸 $\left[\left(\mathrm{CH}_{3} \mathrm{CO}\right)_{2} \mathrm{O} / \mathrm{HNO}_{3}\right]$ 、五氧化二氮 $\left(\mathrm{N}_{2} \mathrm{O}_{5}\right)$ 、四氟 㸴酸硝酰 $\left(\mathrm{NO}_{2} \mathrm{BF}_{4}\right)$ 、硝酸钾/浓硫酸 $\left(\mathrm{KNO}_{3} / \mathrm{H}_{2} \mathrm{SO}_{4}\right)$ 、 $100 \%$ 硝酸/ $\left(100 \% \mathrm{HNO}_{3}\right)$ 作为硝化体系对 FTDO 合成反 应收率的影响, 结果见表 1 所示.

从表 1 可以看出, 以 $100 \% \mathrm{HNO}_{3}$ 作为硝化体系, 效 果最好, 收率可达 99.54\%; 以 $\mathrm{KNO}_{3} / \mathrm{H}_{2} \mathrm{SO}_{4}, \mathrm{NO}_{2} \mathrm{BF}_{4}$, $\mathrm{N}_{2} \mathrm{O}_{5}$ 为硝化试剂，产率分别为 $64.31 \%, 59.46 \%, 48.39 \%$, 
表 1 硝化试剂对化合物 4 收率的影响

Table 1 Effect of nitrogen reagent on the yield of compound 4

\begin{tabular}{lccccc}
\hline Nitration reagent & $\left(\mathrm{CH}_{3} \mathrm{CO}\right)_{2} \mathrm{O} / \mathrm{HNO}_{3}$ & $\mathrm{~N}_{2} \mathrm{O}_{5}$ & $\mathrm{NO}_{2} \mathrm{BF}_{4}$ & $\mathrm{KNO}_{3} / \mathrm{H}_{2} \mathrm{SO}_{4}$ & $100 \% \mathrm{HNO}_{3}$ \\
\hline Yield $\%$ & - & 48.39 & 59.46 & 64.31 & 99.54 \\
Purity $\%$ & - & 90.48 & 94.36 & 66.02 & 99.18 \\
\hline
\end{tabular}

其中 $\mathrm{NO}_{2} \mathrm{BF}_{4}, \mathrm{~N}_{2} \mathrm{O}_{5}$ 为较强的硝化试剂, 使用 $\mathrm{NO}_{2} \mathrm{BF}_{4}$ 为 硝化试剂时, 有目标产物 FTDO 的生成, 产率为 $22.12 \%$; 以 $\mathrm{N}_{2} \mathrm{O}_{5}$ 为硝化试剂时, 有副产物 3-硝基-4-(叔丁基氧化 偶氮基)呋咱的生成，产率为 15.56\%; $\left(\mathrm{CH}_{3} \mathrm{CO}\right)_{2} \mathrm{O} / \mathrm{HNO}_{3}$ 为硝化试剂, 则不能得到产品(用 TLC 监测), 因此, 确 定 $100 \%$ 硝酸为适宜的硝化试剂.

\section{4 成环反应}

\subsection{1 反应温度}

设定反应时间为 $5 \mathrm{~h}$, 试剂料比为 $1: 16$, 考察了反 应温度对 FTDO 收率的影响, 结果见表 2 所示.

表 2 反应温度对 FTDO 产率的影响

Table 2 Effect of reaction temperature on the yield of FTDO

\begin{tabular}{lccccc}
\hline Temperature $/{ }^{\circ} \mathrm{C}$ & 35 & 45 & 55 & 65 & 75 \\
\hline Yield $/ \%$ & - & 35.65 & 46.37 & 43.49 & 30.30 \\
\hline
\end{tabular}

从表 2 可以看出, $35{ }^{\circ} \mathrm{C}$ 时, 反应不完全(用 TLC 监 测); $45{ }^{\circ} \mathrm{C}$ 时，原料点虽然消失，但收率较低为 $36.65 \%$; 温度为 $55{ }^{\circ} \mathrm{C}$ 时, 产率较高为 $46.37 \%$; 温度为 $75{ }^{\circ} \mathrm{C}$ 时, 反应收率有所降低, 其原因可能为温度太高, FTDO 分 解所致, 故 $55^{\circ} \mathrm{C}$ 为最佳的反应温度.

\subsection{2 成环试剂的用量}

设定反应温度为 $55{ }^{\circ} \mathrm{C}$, 反应时间为 $5 \mathrm{~h}$, 考察了试 剂料比对 FTDO 收率的影响, 结果见表 3 所示.

表 3 试剂料比对 FTDO 产率的影响

Table 3 Effect of reagent ratio on the yield of FTDO

\begin{tabular}{ccccc}
\hline $\begin{array}{c}n(\text { compound } 4): \\
n\left(\mathrm{P}_{2} \mathrm{O}_{5}\right)\end{array}$ & $1: 8$ & $1: 12$ & $1: 16$ & $1: 20$ \\
\hline Yield $/ \%$ & - & 39.32 & 46.37 & 42.23 \\
\hline
\end{tabular}

从表 3 可以看出, 试剂料比为 $1: 16$ 时, 合成 FTDO 的收率最高, 为 $46.37 \%$; 当试剂料比为 $1: 8$ 时, 用 TLC 监测反应, 仍有原料存在; 当料比 $1: 12$ 时, 产率为有 $39.32 \%$; 料比为 1:20 时, 产率为 $42.23 \%$; 成环反应的 机理为先酯化, 后进行加成-消去机理, 体系中 $\mathrm{P}_{2} \mathrm{O}_{5}$ 太 少时, 不利于酯化反应的进行, 体系中 $\mathrm{P}_{2} \mathrm{O}_{5}$ 太多时, 又 不利于偏磷酸脂的消去, 因此, 成环反应的最佳料比为 $1: 16$.

\subsection{3 反应溶剂的用量}

设定反应温度为 $55^{\circ} \mathrm{C}$, 反应时间为 $5 \mathrm{~h}$, 试剂料比 为 $1: 16$, 原料化合物 4 为 $1.0 \mathrm{~g}$, 考察了溶剂量对 FTDO 收率的影响, 结果见表 4 .
表 4 溶剂量对 FTDO 产率的影响

Table 4 Effect of the volume of solvent on the yield of FTDO

\begin{tabular}{cccc}
\hline$V / \mathrm{mL}$ & 80 & 160 & 240 \\
\hline Yield/\% & 35.39 & 46.37 & 41.28 \\
\hline
\end{tabular}

从表 4 可以看出，使用 $160 \mathrm{~mL}$ 溶剂时，反应收率最 高为 $46.37 \%$; 而溶剂量为 $80 \mathrm{~mL}$ 时, 由于体系过于粘稠, 搅拌不充分, 收率明显下降，为 $35.39 \%$; 溶剂量 $240 \mathrm{~mL}$ 时, 收率有所下降，为 $41.28 \%$. 在合成 FTDO 时，由于 $\mathrm{P}_{2} \mathrm{O}_{5}$ 反应生成多聚磷酸, 粘度很高, 不利于反应体系的 充分搅拌, 加入适量溶剂既可以使体系充分摚拌, 又不 影响反应的进行.

\section{2 结论}

自行设计了呋咱并 $[3,4-e]-1,2,3,4-$ 四嗪-4,6-二氧化 (FTDO)新的合成路线，以 3,4-二氨基呋咱(DAF)为原料, 经过氧化、缩合、硝化、成环 4 步合成了 FTDO, 总收 率为 $30.89 \%$ (文献 $14.63 \%$ ), 并采用核磁共振、红外、 质谱及元素分析进行了结构表征. 探讨了成环反应的机 理,并研究了硝化及成环反应条件, 确定硝化反应的最 佳条件为: 硝化试剂为 $100 \% \mathrm{HNO}_{3}, 25{ }^{\circ} \mathrm{C}$ 下反应 $2 \mathrm{~h}$, 收 率高达 $99.54 \%$; 成环反应的最佳条件为: $n$ (化合物 4)： $n\left(\mathrm{P}_{2} \mathrm{O}_{5}\right)$ 为 $1: 16$, 溶剂量为 $160 \mathrm{~mL} / \mathrm{g}, 55{ }^{\circ} \mathrm{C}$ 下反应 $5 \mathrm{~h}$, 收率为 $46.37 \%$, 新方法使用了较为廉价, 易得的 $\mathrm{P}_{2} \mathrm{O}_{5}$ 为成环试剂, 具有工业化应用前景.

\section{3 实验部分}

\section{1 主要仪器与试剂}

Nexus 870 傅里叶变换红外光谱仪(美国 Thermo Nicolet 公司); AV500 核磁共振仪(瑞士 Bruker 公司); Vario EL III 元素分析仪; HP5989B 型质谱仪(美国惠普 公司); Varian5000 型高压液相色谱仪(美国 Varian 公司; LC-2010A 型高效液相色谱仪(归一化法)(日本岛津公 司); X-6 型显微熔点测定仪(北京泰克仪器有限公司); Q-200 型差示扫描量热仪(美国 TA 公司); TA2950 热重 仪(美国 Nicolet 公司).

DAF(自制)，双氧水 $(30 \%)$, 硫酸 $(98 \%)$, 纯硝酸，铇 酸钠, 苯, 二氯甲烷, 四氯化碳, 液溴, 氢氧化钠, 叔丁 基胺, 硫代硫酸钠, 氯化亚铜, 五氧化二磷, 均为分析 纯; 乙腈为色谱纯. 


\section{2 实验步骤}

\subsubsection{3-氨基-4-亚硝基呋咱(2)的制备}

搅拌下, 在 $5 \sim 10{ }^{\circ} \mathrm{C}$ 将 $200 \mathrm{~mL}$ 苯, $145 \mathrm{~mL}$ (1290 $\mathrm{mmol}) 30 \%$ 双氧水和 $16.5 \mathrm{~g}(50 \mathrm{mmol})$ 铇酸钠依次加入烧 瓶中, 滴加 $10 \mathrm{~mL}$ 浓硫酸 $(180 \mathrm{mmol})$, 加完后分批加入 5 $\mathrm{g}(50 \mathrm{mmol})$ 化合物 1 , 升温至 $10 \sim 15{ }^{\circ} \mathrm{C}$ 反应 $1.5 \mathrm{~h}$. 分离 有机相, 向水相加入 $100 \mathrm{~mL}$ 苯, 室温下继续反应 $1 \mathrm{~h}$, 分离有机相, 与前一步的有机相合并, 过滤. 水洗有机 相, 用无水硫酸镁干燥, 蒸馏除去溶剂得到土黄色固体 $5.19 \mathrm{~g}$, 收率为 $91.05 \%$. m.p. $76 \sim 78{ }^{\circ} \mathrm{C} ;{ }^{1} \mathrm{H}$ NMR (DMSO- $\left.d_{6}, 500 \mathrm{MHz}\right) \delta: 6.86$ (s, 2H); IR (KBr) $v: 3421$, 3318, 1628, 1531, 1482, 1409, 1284, $1014 \mathrm{~cm}^{-1}$. Anal. calcd for $\mathrm{C}_{2} \mathrm{H}_{2} \mathrm{~N}_{4} \mathrm{O}_{2}$ : C 21.06, H 1.77, N 49.12; found C 20.82, H 1.77, N 48.95

\subsubsection{3-氨基-4-(叔丁基氧化偶氮基)呋咱(3)的合成}

在 $0{ }^{\circ} \mathrm{C}$ 下, 依次将 $150 \mathrm{~mL} 10 \%$ 的氢氧化钠水溶液, $24.0 \mathrm{~g}$ (150 mmol)液溴, $7.5 \mathrm{~g}(100 \mathrm{mmol})$ 叔丁基胺和 200 $\mathrm{mL}$ 二氯甲烷加入烧瓶中, 反应 $5 \mathrm{~h}$. 将反应液倒入冰水 中, 分离有机相, 水相用二氯甲烷萃取. 合并有机相, 水洗至中性, 转移到烧瓶中, 然后将 $10.0 \mathrm{~g}(100 \mathrm{mmol})$ 的 $\mathrm{CuCl}, 500 \mathrm{~mL}$ 二氯甲烷依次加入烧瓶中, 再分批加入 $5.7 \mathrm{~g}(50 \mathrm{mmol})$ 化合物 2 . 然后在 $15 \sim 25{ }^{\circ} \mathrm{C}$ 反应 $15 \mathrm{~h}$. 将 反应液倒入冰水中, 加入硫代硫酸钠, 过滤, 分离有机 相, 水相用二氯甲烷萃取 $(50 \mathrm{~mL} \times 4)$, 合并有机相, 水 洗, 加入无水氯化钻干燥, 烝馏除去溶剂, 得到棕黄色 固体 $6.8 \mathrm{~g}$, 收率 $73.50 \%$. m.p. $156 \sim 158{ }^{\circ} \mathrm{C} ;{ }^{1} \mathrm{H}$ NMR (DMSO- $\left.d_{6}, 500 \mathrm{MHz}\right) \delta: 1.43(\mathrm{~s}, 9 \mathrm{H}), 6.59(\mathrm{~s}, 2 \mathrm{H}) ;{ }^{13} \mathrm{C}$ NMR (DMSO- $\left.d_{6}, 500 \mathrm{MHz}\right) \delta: 25.06,59.99,150.77$, 151.55; IR (KBr) v: 3472, 3356, 2980, 1624, 1566, 1453, 1367, $1183 \mathrm{~cm}^{-1}$; MS (EI) $\mathrm{m} / z(\%): 185\left(\mathrm{M}^{+}, 5\right), 112(20)$, 102 (20), 57 (100), 41 (35), 29 (28). Anal. calcd for $\mathrm{C}_{6} \mathrm{H}_{11} \mathrm{~N}_{5} \mathrm{O}_{2}$ : C 38.92, H 5.99, N 37.82; found C 39.36, H 5.96, N 35.60.

3.2.3 3-硝氨基-4-(叔丁基氧化偶氮基)呋咱(4)的合 成

在 $0{ }^{\circ} \mathrm{C}$ 下, 将 $2.72 \mathrm{~g}$ 纯硝酸滴加到 $2 \mathrm{~g}(10.8 \mathrm{mmol})$ 化合物 3 的四氯化碳 $(40 \mathrm{~mL})$ 溶液中, 加完后低温反应 $0.5 \mathrm{~h}$, 然后在 $25{ }^{\circ} \mathrm{C}$ 反应 $2 \mathrm{~h}$. 将反应液蒸干, 加入 100 $\mathrm{mL}$ 二氯甲烷提取, 水洗, 水相用二氯甲烷萃取 (50 $\mathrm{mL} \times 2$ ), 合并有机相, 加入无水硫酸镁干燥, 蒸馏除去 溶剂, 得到黄色固体 $2.47 \mathrm{~g}$, 收率 99.54\%. m.p. 92 94 ${ }^{\circ} \mathrm{C} ;{ }^{1} \mathrm{H}$ NMR $\left(\mathrm{CDCl}_{3}, 500 \mathrm{MHz}\right) \delta: 1.52(\mathrm{t}, J=64 \mathrm{~Hz}, 9 \mathrm{H})$, $11.34(\mathrm{~s}, 1 \mathrm{H}) ;{ }^{13} \mathrm{C}$ NMR $\left(\mathrm{CDCl}_{3}, 500 \mathrm{MHz}\right) \delta: 25.34$, 61.90, 142.91, 150.35; IR (KBr) v: 3291, 2983, 1621, $1510,1488,1315,1164 \mathrm{~cm}^{-1}$; MS (EI) $\mathrm{m} / \mathrm{z}(\%): 230\left(\mathrm{M}^{+}\right.$,
40), 212 (97), 172 (35), 154 (28), 119 (18), 98 (20). Anal. calcd for $\mathrm{C}_{6} \mathrm{H}_{1} \mathrm{~N}_{6} \mathrm{O}_{3}$ : C 31.30, H 4.35, N 36.52; found $\mathrm{C}$ $31.73, \mathrm{H} 4.41, \mathrm{~N} 36.12$.

3.2.4 呋咱并 $[3,4-e]-1,2,3,4-$ 四嗪-4,6-二氧化(FTDO) 的合成

在 $0{ }^{\circ} \mathrm{C}$ 下, 将 $1 \mathrm{~g}$ (4.35 mmol)化合物 4 的乙腈 $(160$ $\mathrm{mL})$ 溶液滴加到五氧化二磷 $(20 \mathrm{~g})$ 中, 低温反应 $0.5 \mathrm{~h}$, 然 后在 $55{ }^{\circ} \mathrm{C}$ 反应 $5 \mathrm{~h}$. 将反应液倒入 $200 \mathrm{~mL}$ 冰水中, 用 $10 \mathrm{~mL} \times 20$ 二氯甲烷萃取, 合并有机相, 加入无水硫酸 镁干燥, 蒸馏除去溶剂, 得到黄色油状物 $0.70 \mathrm{~g}$, 脱色 (活性白土), 蒸馏除去溶剂, 得到亮黄色晶体 $0.315 \mathrm{~g}$, 产率为 46.37\%. m.p. 109 $111{ }^{\circ} \mathrm{C} ;{ }^{13} \mathrm{C}$ NMR (acetone- $d_{6}$, $500 \mathrm{MHz}) \delta$ : $156.81,144.71 ;{ }^{14} \mathrm{~N}$ NMR (acetone- $d_{6}$, $\mathrm{MeNO}_{2}$ as the standard, $\left.500 \mathrm{MHz}\right) \delta: 37.6\left(v_{1 / 2}=519 \mathrm{~Hz}\right.$, $\mathrm{N}-1,3), 10.0\left(v_{1 / 2}=458 \mathrm{~Hz}, \mathrm{~N}-5\right),-42.2\left(v_{1 / 2}=9 \mathrm{~Hz}\right.$, $\mathrm{N}-6),-50.7\left(v_{1 / 2}=9 \mathrm{~Hz}, \mathrm{~N}-4\right),-104.7\left(v_{1 / 2}=279 \mathrm{~Hz}\right.$, $\mathrm{N}-7) ;{ }^{15} \mathrm{~N}$ NMR (acetone- $d_{6}, \mathrm{MeNO}_{2}$ as the standard, 500 MHz) $\delta: 35.6,38.8(\mathrm{~N}-1,3),-42.3(\mathrm{~N}-6),-50.9(\mathrm{~N}-4)$, $-104.7(\mathrm{~N}-7) ;{ }^{17} \mathrm{O}$ NMR (acetone- $d_{6}, \mathrm{MeNO}_{2}$ as the standard, $500 \mathrm{MHz}) \delta: 532.3\left(v_{1 / 2}=251 \mathrm{~Hz}\right), 504.2\left(v_{1 / 2}=186\right.$ Hz), $441.7\left(v_{1 / 2}=441 \mathrm{~Hz}\right)$; IR (KBr) $v: 1589,1543,1514$, 1460, 1418, 1317, 1148, 1018, $939 \mathrm{~cm}^{-1}$; MS (EI) $\mathrm{m} / z(\%)$ : $156\left(\mathrm{M}^{+}, 25\right), 84$ (4), 68 (45), 52 (13), 30 (100). Anal. calcd for $\mathrm{C}_{2} \mathrm{~N}_{6} \mathrm{O}_{3}$ : C 15.38, N 53.47; found $\mathrm{C} 15.38, \mathrm{~N}$ 53.85 .

\section{References}

[1] Dong, H.-S. Chin. J. Energ. Mater. 2004, (supplement), 1 (in Chinese). (董海山, 含能材料, 2004, (增刊), 1.)

[2] Huang, M.; Li, H.-Z.; Dong, H.-S. In 2004 the National Energetic Materical Development an Application Conference, Xiamen, 2004, p. 73 (in Chinense)

(黄明, 李洪珍, 董海山, 2004 全国含能材料发展与应用学术研 讨会, 厦门, 2004, p. 73.)

[3] Li, J.-R. Chin. J. Explos. Propellants 1998, 3, 56 (in Chinese). (李加荣, 火炸药学报, 1998, 3, 56.)

[4] Hiskey, M.; Chavez, D. In 27th International Pyrotechnics Seminar, Colorado, 2000, pp. $3 \sim 14$.

[5] Hiskey, M. A.; Chavez, D. E.; Naud, D. L. NTIS No: DE-2001776133, 2001.

[6] Wu, Y.-H. Organic Chemistry, University of Science and Technology of China, Hefei, 2002 (in Chinese). (伍越睘, 有机化学, 中国科技大学出版社, 合肥, 2002.)

[7] Yue, S.-T. Ph.D. Dissertation, National University of Defense Technology, Changsha (in Chinese). (岳守体, 博士论文, 国防科技大学, 长沙, 2003)

[8] Xu, S.-L. Ph.D. Dissertation, National University of Defense Technology, Changsha (in Chinese). (徐松林, 博士论文, 国防科技大学, 长沙, 2005.)

[9] Aleksandr, M. C. MendeLeev Commun. 1995, 5(6), 227.

[10] Lempert, D. B.; Nechiporenko, G. N. Khim. Fiz. 2004, 23(5), 75. 
[11] Kiselev, V. G.; Gritsan, N. P. Combust. Explos. Shock Waves 2007, 43(5), 562.

[12] Kalmykov, P. I.; Burtsev, Y. A. In Proceeding of the 3th All-Russian Conference Energetic Condensed Systems (Chernogolovka), Yaunus, Moscow, 2004, pp. 64 66 .

[13] Li, H.-Z.; Zhou, X.-Q. Chin. J. Org. Chem. 2008, 28, 1646 (in Chinese).

(李洪珍, 周小清, 有机化学, 2008, 28, 1646.)

[14] Apasove, E. T.; Dzhetigenov, B. A. Russ. Chem. Bull. 1991, 1234.

[15] Apasov, E. T.; Sheremetev, A. B. Russ. Chem. Bull. 1993, 1500.

[16] Tatyana, M. M. MendeLeev Commun. 2001, 11(1), 30.

[17] Nelson, V.; Srianz, A. J. Org. Chem. 1976, 4l(10), 1751.
[18] Aleksandr, M. C.; Sergei, E. S. Mendeleev Commun. 1995, 5(3), 102.

[19] Frumkin, A. E.; Churakov, A. M. Russ. Chem. Bull. 2000, 49(3), 482.

[20] Silverstein, R. M.; Webster, F. X. Spectrometric Identification of Organic Compounds, 7th ed., Trans. by WuXi AppTec Co. Ltd. Analysis Division, East China University of Science and Technology Press, Shanghai, 2007, pp. 44 69, 71 105 (in Chinese).

(西尔弗斯坦，韦伯斯特，有机化合物的波谱解析，第七版，药明 康德新药开发有限公司分析部译, 华东理工大学出版社, 上海, 2007, pp. 44 69, 71 105.)

(Cheng, F.) 